\title{
Influence of surface roughness on diffraction in the externally occulted Lyot solar coronagraph Application to ASPIICS
}

\author{
R. Rougeot ${ }^{1,2}$, R. Flamary ${ }^{1}$, D. Mary ${ }^{1}$, and C. Aime ${ }^{1}$ \\ ${ }^{1}$ Laboratoire Lagrange, Université Côte d'Azur, Centre National de la Recherche Scientifique, Observatoire de la Côte d'Azur, \\ Parc Valrose, 06108 Nice, France \\ e-mail: raphael.rougeot@esa.int \\ 2 European Space Research and Technology Center, European Space Agency, Keplerlaan 1, 2201 Noordwijk, The Netherlands
}

Received 13 November 2018 / Accepted 14 March 2019

\begin{abstract}
Context. The solar coronagraph ASPIICS will fly on the future ESA formation flying mission Proba-3. The instrument combines an external occulter of diameter $1.42 \mathrm{~m}$ and a Lyot solar coronagraph of $5 \mathrm{~cm}$ diameter, located downstream at a distance of $144 \mathrm{~m}$. Aims. The theoretical performance of the externally occulted Lyot coronagraph has been computed by assuming perfect optics. In this paper, we improve related modelling by introducing roughness scattering effects from the telescope. We have computed the diffraction at the detector, that we compare to the ideal case without perturbation to estimate the performance degradation. We have also investigated the influence of sizing the internal occulter and the Lyot stop, and we performed a sensitivity analysis on the roughness.

Methods. We have built on a recently published numerical model of diffraction propagation. The micro-structures of the telescope are built by filtering a white noise with a power spectral density following an isotropic ABC function, suggested by Harvey scatter theory. The parameters were tuned to fit experimental data measured on ASPIICS lenses. The computed wave front error was included in the Fresnel wave propagation of the coronagraph. A circular integration over the solar disk was performed to reconstruct the complete diffraction intensity.

Results. The level of micro-roughness is $1.92 \mathrm{~nm}$ root-mean-square. Compared to the ideal case, in the plane of the internal occulter, the diffraction peak intensity is reduced by $\simeq 0.001 \%$. However, the intensity outside the peak increases by $12 \%$ on average, up to $20 \%$ at $3 R_{\odot}$, where the mask does not filter out the diffraction. At detector level, the diffraction peak remains $\simeq 10^{-6}$ at $1.1 R_{\odot}$, similar to the ideal case, but the diffraction tail at large solar radius is much higher, up to one order of magnitude. Sizing the internal occulter and the Lyot stop does not improve the rejection, as opposed to the ideal case.

Conclusions. Besides these results, this paper provides a methodology to implement roughness scattering in the wave propagation model for the solar coronagraph.
\end{abstract}

Key words. Sun: corona - instrumentation: high angular resolution - methods: numerical

\section{Introduction}

The study of the corona of the Sun in white light requires highcontrast, as the coronal brightness in this spectral band ranges from $10^{-6}$ to $10^{-10}$ with respect to the mean solar brightness (Cox 2000). Perfect eclipse conditions are thus needed to prevent the sunlight from blinding the observation. Moreover, high angular resolution is also a key aspect - in the order of the arcsecond, when aiming to capture fine coronal structures or Coronal Mass Ejections (Zhukov et al. 2000; Peter et al. 2013).

The first actual observation of the corona of the Sun in the absence of a natural eclipse was performed by the French astronomer Bernard Lyot (Lyot 1939). The basic principle of his novel concept consisted in focusing the solar image on a mask set in the focal plane of the telescope, while letting the coronal light propagate further. From this, solar coronagraphy was further developed with the external occultation technique introduced by Evans (1948), and the use of serrated or multiple disks (Purcell \& Koomen 1962; Newkirk \& Bohlin 1965). Koutchmy
(1988) gives a review of the early advent of spaceborne solar coronagraphy and associated development.

The future ESA Formation Flying mission Proba-3 will fly the advanced solar coronagraph ASPIICS (Association de Satellites Pour l'Imagerie et l'Interférométrie de la Couronne Solaire; Lamy et al. 2010; Renotte et al. 2015; Galano et al. 2018). The novelty of this instrument is that it is split over the two spacecrafts. The occulter separation and diameter reach unprecedented dimensions for a space coronagraph, reducing the size of the vignetted zone, close to the solar limb. The Occulter Spacecraft will carry a large external occulting disk of diameter $1.42 \mathrm{~m}$, while the telescope, with an aperture of $5 \mathrm{~cm}$ diameter, is located downstream at a distance of $144 \mathrm{~m}$, in the Coronagraph Spacecraft. The complete optical system is a hybrid Lyot-style solar coronagraph, described in Galy et al. (2015).

Performance in coronagraphy is mostly driven by straylight, in particular the residual diffracted light of the bright source. Dedicated analysis is required to predict the level of contrast that can be achieved by the instrument, which also governs its design. 
The estimation of diffraction produced by the edge of an (external) occulter remains a delicate problem. One first approach relies on experimental measurements, as in the work of Bout et al. (2000) for the LASCO C2 coronagraph (Brueckner et al. 1995), or measurements on the real sky with a small-scale model as done by Venet et al. (2010) for the ASPIICS coronagraph. However, the very large size of the latter instrument prevents us from performing any full-scale experimental characterization. Landini et al. (2010) propose a scaled setup to measure the diffraction produced by the edge of occulters of various shapes. This second approach consists of numerical computations based on the Fresnel diffraction theory (Born \& Wolf 2006). In stellar coronagraphy, this subject has been very prolific and resulted in optimized shapes of external occulters (Cash 2006; Vanderbei et al. 2007; Cady 2012). The case of solar coronagraphy brings the additional difficulty that the Sun is an extended light source. The work of Lenskii (1981) can be held as an example, and more recently Verroi et al. (2008), Aime (2013), Rougeot \& Aime (2018) studied the solar case with ASPIICS dimensions.

As described above, several studies investigated the computation of the diffraction by an external occulter on the one hand. On the other hand, the light propagation of the wave fronts through the entire coronagraphic system is a complementary problem that must be solved in order to estimate the intensity of the residual diffracted sunlight at the detector. Aime et al. (2002) study this problem for the classic Lyot coronagraph. Rougeot (2017) propose a model for the case of the hybrid Lyot-style solar coronagraph. These computations all rely on the strong assumptions that the optics are ideal, and the system is perfectly centered and co-aligned. Therefore, the demonstrated straylight rejection remains theoretical and should be considered as a best case scenario. The degradation of performance when deviating from such an ideal case must be addressed. As an example, Shestov \& Zhukov (2018) treated the problem of misalignments and tilts between the coronagraph and the external occulter for ASPIICS, and how they influences the intensity and the spatial distribution of the diffraction on the detector. Rougeot et al. (2018) report an overview of the different diffraction models and computations made for ASPIICS.

In this paper, we present the study of a specific type of perturbation, that is the scattering of light induced by surface roughness. We built upon the model of Rougeot (2017), which we improved by implementing the wave-front error induced by surface micro-structures, following the theory of Harvey et al. (2007). Our analysis focuses on the telescope, that is, the first optical element, as it is known to be the most stringent for straylight in coronagraphy (Brueckner et al. 1995). To better represent the ASPIICS system, the modeled micro-structures were derived by fitting the actual model used in the optical design of ASPIICS coronagraph. Furthermore, our paper aims to provide a practical method with which to implement the wave perturbation in the light propagation model.

The paper is organized as follows. Section 1 contains this introduction. In Sect. 2 we introduce the model of diffraction and light propagation. Section 3 presents the numerical implementation of the roughness scattering in the diffraction model. Results are discussed in Sect. 4. Conclusions are given in Sect. 5.

\section{Presentation of the propagation model}

In this section we summarize the propagation model for the externally occulted Lyot-style solar coronagraphe. All notations are as in Rougeot (2017), and we refer the reader to this paper for further details.

\subsection{Description of the coronagraph}

The coronagraph is made of six successive key planes denoted as $O, A, B, O^{\prime}, C$, and $D$. Figure 1 illustrates the system - not to scale. The external occulter is modeled as a perfect sharp-edged disk of radius $R=710 \mathrm{~mm}$ located in plane $O$. We note that the real occulter for ASPIICS will have a toroidal shape. Plane $A$ is positioned at a distance $z_{0}=144.348 \mathrm{~m}$ downstream, where the entrance aperture of radius $R_{\mathrm{p}}=25 \mathrm{~mm}$ is set. The sun, at infinity, has an angular radius of $R_{\odot}=0.0046542 \mathrm{rad}$, being $\approx 16.2$ arcmin. Therefore, the geometric radius of the external occulter, as viewed from the centre of the pupil, is 1.0568 times the size of the sun. We have assumed that the primary objective L1 of focal length $f_{1}=330.348 \mathrm{~mm}$ coincides with the pupil. Plane $B$ is the focal plane, where the image of the sun is focused in the absence of external occulter. In the classical Lyot coronagraph, the Lyot mask is set there (Aime et al. 2002). Plane $O^{\prime}$ denotes the image plane of plane $O$ made by the primary objective. It is located at a distance

$z_{1}=\frac{z_{0} \times f_{1}}{z_{0}-f_{1}}$

from plane $A$. Equation (1) is simply derived from the thin lens formula. We have $z_{1}=331.106 \mathrm{~mm}$, so plane $O^{\prime}$ is further than plane $B$. The internal occulter is set in the plane $O^{\prime}$, because this is where the diffracted light from the edge of the external occulter is focused (Rougeot 2017). The second objective L2 of focal $f_{2}$ is also located at plane $O^{\prime}$. Plane $C$ consists of the image of plane $A$ made by L2, located at $z_{2}$ from plane $O^{\prime}$. The Lyot stop and the final relay lenses L 3 of effective focal length $f_{3}$ are in this plane. Finally, plane $D$ is the final focal plane where the detector is located.

The internal occulter is modeled as a simple disk of radius $R_{\text {io }}$. Because plane $O^{\prime}$ is the conjugate of plane $O$, related unit system is preferably in terms of unit of external occulter image. However, it is more convenient and understandable to speak in terms of angular or metric units. A simple proportionality rule gives the relation between the different units - from $R, z_{0}$ and $z_{1}$. In our study, $R_{\text {io }}=1.662 \mathrm{~mm}$, which corresponds to $1.079 R_{\odot}$ or to 1.021 times the image of the radius of the external occulter. Additionally, there is a circular hole inside the internal occulter, in order to image LEDs positioned on the external occulter this optical metrology is described in Loreggia (2015). Its radius is fixed to $0.489 \mathrm{~mm}$, about $30 \%$ of $R_{\text {io }}$. The size of the Lyot stop $R_{\mathrm{ls}}$ is expressed in units of pupil. In our study, we fixed $R_{\mathrm{ls}}=0.97 R_{\mathrm{p}}$, which gives $R_{\mathrm{ls}}=24.25 \mathrm{~cm}$.

\subsection{Analytical framework}

We set a Cartesian reference frame $(x, y, z)$, where the $z$-axis defines the optical axis. We also defined the transverse radius $r=\sqrt{x^{2}+y^{2}}$ and polar angle $\theta$, i.e., $x=r \cos \theta$ and $y=r \sin \theta$. In the following, both notations will be used. A point source is located in the sky by two angular coordinates $(\alpha, \beta)$, which refer to the azimuth and elevation, respectively. The sun is modeled as a disk made of a collection of point sources, and its center lies on the $z$-axis. We used the same center-to-limb variations function $B_{\odot}$ as in Rougeot (2017) to model the brightness of the solar disk, which was derived from van Hamme (1993).

Our study focuses on monochromatic light at the wavelength $\lambda=550 \mathrm{~nm}$, to be representative of the spectral band-pass of 
R. Rougeot et al.: Influence of surface roughness on diffraction in the externally occulted Lyot solar coronagraph

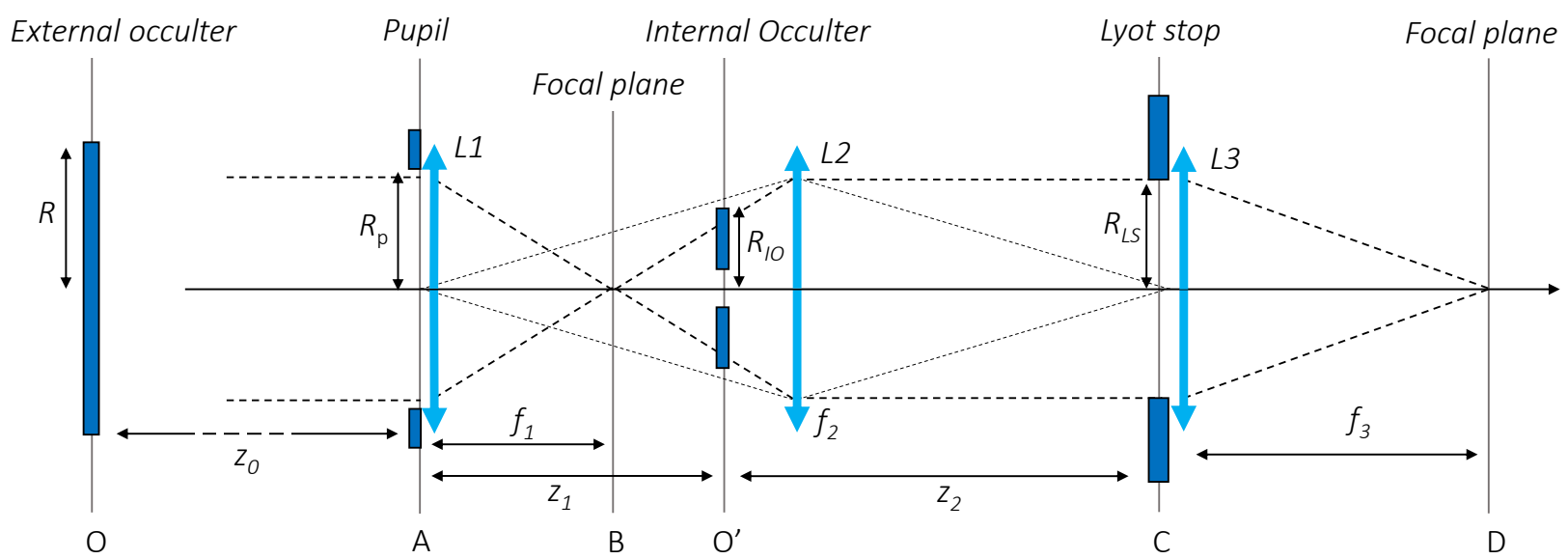

Fig. 1. Schematic representation of the hybrid externally occulted Lyot solar coronagraph, adapted from Rougeot (2017) - Figure not to scale. The six key planes $O, A, B, O^{\prime}, C$, and $D$, and key geometrical parameters are reported.The dotted lines illustrate geometrical optical paths. The variables are as follows: $R$ radius of the external occulter; $z_{0}, z_{1}$ and $z_{2}$ the distances from plane $O$ to $A$, plane $A$ to $O^{\prime}$ and plane $O^{\prime}$ to $C$, respectively; $R_{\mathrm{p}}$ radius of the entrance pupil; $f_{1}, f_{2}$, and $f_{3}$ the focal lengths of the objectives L1, L2, and L3, respectively; $R_{\mathrm{io}}$ radius of the internal occulter; $R_{\mathrm{ls}}$ radius of the Lyot stop. See text for the detailed description.

ASPIICS [540 nm, $570 \mathrm{~nm}]$. We expect no significant difference in the spatial distribution of observed diffraction intensities over the spectral width $\Delta \lambda=30 \mathrm{~nm}$. The reason is the following. Aime (2013) studied the diffraction pattern behind the external occulter from the on-axis point source, known as the Arago spot. The author showed that the central bright spot has a width of the order of $\simeq 150 \lambda$. For $\lambda=550 \mathrm{~nm}$, the width is $82.5 \mu \mathrm{m}$, and a variation of $4.5 \mu \mathrm{m}$ across ASPIICS spectral range. In the case of solar observations, the point source diffraction pattern is convolved with the stenope image of the Sun (see Eq. (8) later), which has a radius of $671 \mathrm{~mm}$ in the geometry of ASPIICS (Rougeot \& Aime 2018). Therefore, the small variation of the spatial distribution of the diffraction with the wavelength becomes negligible when performing such integration, and a monochromatic study remains sufficient. We note that the influence of the wavelength is different for the stellar case.

In the geometry of ASPIICS, the Fresnel number $N_{f}=$ $R^{2} / \lambda z_{0}$ is about 6400 , so the diffraction by a thin sharp-edged disk is correctly described by the Fresnel regime. This problem for long baseline solar coronagraph has been treated by Aime (2013). Light propagation is based on Fresnel free-space propagation along the $z$-axis (Born \& Wolf 2006). In a given transverse plane $\mathcal{K}$, the scalar wave front is represented by its complex amplitude $\Psi_{\mathcal{K}}(x, y)$. For a point source awith coordinates $(\alpha, \beta)$ in the sky, the complex amplitude of the diffracted wave front in plane $A-$ at a distance $z_{0}$ - is given by

$\Psi_{A, \alpha, \beta}(x, y)=Q_{\alpha, \beta} \times T_{\alpha, \beta}(x, y) \times \Psi_{A, 0,0}\left(x+\alpha z_{0}, y+\beta z_{0}\right)$,

where

$T_{\alpha, \beta}(x, y)=\exp \left(-2 i \pi \frac{\alpha x+\beta y}{\lambda}\right)$

$Q_{\alpha, \beta}=\exp \left(-i \pi \frac{\left(\alpha^{2}+\beta^{2}\right) z_{0}}{\lambda}\right)$

(Offset)

$\Psi_{A, 0,0}(x, y)=1-\frac{1}{i \lambda z_{0}} \iint_{\eta^{2}+\xi^{2} \leq R^{2}} \exp \left(i \pi \frac{(x-\xi)^{2}+(y-\eta)^{2}}{\lambda z_{0}}\right) \mathrm{d} \xi \mathrm{d} \eta$,

$\Psi_{A, 0,0}$ is the complex amplitude of the diffracted wave front from the on-axis point source. It is computed here as a radial Hankel transformation, but alternative methods can be implemented, such as the Lommel series (Aime 2013), or the Maggi-Rubinowicz representation (Rougeot \& Aime 2018). The intensity $\left|\Psi_{A, 0,0}\right|^{2}$ produces the famous Arago bright spot.

The light propagation inside the coronagraph is based on paraxial Fourier optics (Goodman 2005) from one plane to the next one. We refer to the radial transmission functions of the entrance pupil in plane $A$, the internal occulter in plane $O^{\prime}$, and the Lyot stop in plane $C$ as $\mathcal{P}(r), \mathcal{M}(r)$, and $\mathcal{L}(r)$, respectively. The wave fronts $\Psi_{B, \alpha, \beta}, \Psi_{O^{\prime}, \alpha, \beta}, \Psi_{C, \alpha, \beta}$ and $\Psi_{D, \alpha, \beta}$, in the planes $B, O^{\prime}, C$, and $D$, respectively, are computed following the model of Rougeot (2017)

$$
\begin{aligned}
\Psi_{B, \alpha, \beta}(x, y) & =\frac{1}{i \lambda f_{1}} \times \mathcal{F}_{\lambda f_{1}}\left[\Psi_{A, \alpha, \beta} \times \mathcal{P}\right] \\
\Psi_{O^{\prime}, \alpha, \beta}(x, y) & =\frac{1}{i \lambda z_{1}} \times \mathcal{F}_{\lambda z_{1}}\left[\Psi_{A, \alpha, \beta} \times \mathcal{P} \times \varphi_{-z_{0}}\right] \\
\Psi_{C, \alpha, \beta}(x, y) & =\frac{1}{i \lambda z_{2}} \times \mathcal{F}_{\lambda z_{2}}\left[\Psi_{O^{\prime}, \alpha, \beta} \times \mathcal{M}\right] \times \varphi_{+z_{0}}(r) \\
\Psi_{D, \alpha, \beta}(x, y) & =\frac{1}{i \lambda f_{3}} \times \mathcal{F}_{\lambda f_{3}}\left[\Psi_{C, \alpha, \beta} \times \mathcal{L}\right]
\end{aligned}
$$

where $\varphi_{z}(r)=\exp \left(i \pi r^{2} / \lambda z\right)$, and $r=\sqrt{x^{2}+y^{2}}$ is the transverse radius, and $z_{2}$ is the distance from plane $O^{\prime}$ to plane $C$. We note that in the model, the actual values of $f_{2}$ and $z_{2}$ are not relevant, because plane $C$ is the image of plane $A$, and $f_{3}$ is chosen equal to $f_{1}$ for simplicity. $\mathcal{F}_{\lambda z}$ denotes the $2 \mathrm{D}$ Fourier transformation with spatial frequencies $u=x / \lambda z$ and $v=y / \lambda z$. In Eqs. (4)-(7), we simplified the quadratic phase factors that should remain in front of the Fourier transformations, by assuming that the optical layout is such that they cancel from one plane to to the next one (Aime et al. 2002). We note that, in Eq. (5), the quadratic phase factor $\varphi_{-z_{0}}$ results from the propagation from plane $A$ to $O^{\prime}$, instead of plane $B$. The factor $\varphi_{+z_{0}}$ in Eq. (6) cancels this factor, in order to retrieve the exact image of plane $A$ in plane $C$. Again, we refer the reader to Rougeot (2017) for the detailed reasoning and derivation.

To compute the integrated intensities over the solar disk, for convenience we changed to polar coordinates $(\rho, \phi)$ in place of $(\alpha, \beta)$, i.e., $\alpha=\rho \cos \phi$ and $\beta=\rho \sin \phi$. The intensity $I_{\mathcal{K}}$ in the plane $\mathcal{K}$ results from the integration over all the point source responses, weighted by the limb darkening $B_{\odot}(\rho)$ written as a 
radial function.

$I_{\mathcal{K}}(x, y)=G_{\mathcal{K}} \times \int_{0}^{R_{\odot}} \int_{0}^{2 \pi} B_{\odot}(\rho) \times\left|\Psi_{\mathcal{K}, \rho, \phi}(x, y)\right|^{2} \rho \mathrm{d} \rho \mathrm{d} \phi$

where $\mathcal{G}$ is a normalization constant - discussed below, and $\mathcal{K}=A, B, O^{\prime}, C$, or $D$. To compute Eq. (8), 1000 points along a solar radius are used in the simulation, which defined $\mathrm{d} \rho$, and a circular integration method is used, as described in Rougeot (2017).

The numerical computation of the wave fronts in the successive planes is performed using standard two dimensional FFT routines in Matlab. Large arrays of size $N \times N$ are used, to perform zero padding. To derive the spatial sampling $s_{A}$ in plane $A$, we followed the approach of Rougeot (2017, Sect. 4.1 and Eq. (13)). The reasoning is to set the same number of points in the pupil radius $R_{\mathrm{p}}$ in plane $A$ and in the image of the external occulter in plane $O^{\prime}$.

Particular care must be taken regarding the normalization for the computed intensities. In Rougeot (2017), a systematic normalization was performed with respect to the raw image of the solar disk, computed for every plane $\mathcal{K}$. This method had the strong disadvantage of requiring additional computations without the coronagraphic masks. For this study, we improved the implementation such that the flux which propagates is conserved from one plane to the next one. First, we imposed that the integrated intensity over the pupil for a single point source is equal to 1 . Second, since the elementary solar sources have a certain angular extent, given by $\rho \mathrm{d} \rho \mathrm{d} \phi$ (or similarly $\mathrm{d} \alpha \mathrm{d} \beta$ ), the étendue must also be conserved. The intensity after propagation through the plane $A$ must be scaled by the solid angle element $\left(\lambda / N s_{a}\right)^{2}$. Finally, from plane $C$ to plane $D$, the Lyot stop actually reduces the throughput by $R_{\mathrm{ls}}^{2}$. We thus derived

$\mathcal{G}_{\mathcal{K}}=\frac{1}{\pi R_{\mathrm{p}}^{2}} \times\left(\lambda / N s_{a}\right)^{-2}$ for $\mathcal{K}=B, O^{\prime}, C$

$\mathcal{G}_{D}=\frac{1}{\pi R_{\mathrm{p}}^{2}} \times\left(\lambda / N s_{a}\right)^{-2} \times \frac{1}{R_{\mathrm{ls}}^{2}}$.

The results of the computation reported in this paper have been made using a machine two 14 core Intel Xeon processors and $512 \mathrm{~GB}$ of RAM. We used $N=2^{13}=8192$, which gives $s_{A}=18.5 \mu \mathrm{m}$. The diameter of pupil $P(r)$ in plane $A$ is thus $33 \%$ of the total spatial field of $15.6 \mathrm{~cm}$. Table 1 recaps the parameters of the study.

We validated our improved model by reproducing the results of Rougeot (2017). As a reference for the study, Figure 2 plots the radial integrated intensity $I_{D}(r)$ in plane $D$, in logarithmic scale, computed for the ideal case, that is, without any perturbation. The red curve shows the residual diffracted sunlight, with a $10^{-6}$ diffraction fringe located at $r \simeq 1.1 R_{\odot}$, which corresponds to the size of the internal occulter. Diffracted light decreases rapidly with $r$ but remains at a level higher than $10^{-11}$. As a comparison, the K-corona ranges from $10^{-6}$ to $10^{-9}$ in $\left[1 R_{\odot} ; 3 R_{\odot}\right]$. The black curve shows the raw image of the Sun, correctly normalized to one at its center, which validates our coefficient $\mathcal{G}$.

\section{Model of scattering from surface roughness}

Surface roughness of polished lenses undeniably induce scattering, which is a diffraction process caused by the micro-structures of the surface. The light is then scattered away from the specular direction. In optics engineering, the scattered light behavior is usually described by the Bi-dimensional transmission
Table 1. Main parameters of the numerical study - based on ASPIICS.

\begin{tabular}{ll}
\hline \hline Parameter & Value \\
\hline Wavelength & $\begin{array}{l}\lambda=550 \mathrm{~nm} \\
R_{\odot}=0.0046542 \mathrm{rad} \\
=960 \mathrm{arcsec}\end{array}$ \\
Angular radius of the Sun & $\begin{array}{l}R=710 \mathrm{~mm} \\
\end{array}$ \\
Radius of the external occulter & $\begin{array}{l}z_{0}=144.348 \mathrm{~m} \\
R_{\mathrm{p}}=25 \mathrm{~mm}\end{array}$ \\
Distance plane $O-$ plane $A$ & $f_{1}=330.348 \mathrm{~mm}$ \\
Fadius of the pupil & $R_{\mathrm{io}}=1.662 \mathrm{~mm}$ \\
Radius of the internal occulter & $=1.079 R_{\odot}$ \\
& $0.489 \mathrm{~mm}$ \\
Hole radius of the internal occulter & $R_{\mathrm{ls}}=0.97 R_{\mathrm{p}}$ \\
Radius of the Lyot stop & $N=24.25 \mathrm{~cm}$ \\
& $N=2^{13}=8192$ \\
Size of the array for FFT & $s_{A}=18.5 \mu \mathrm{m}$ \\
Sampling in plane $A$ &
\end{tabular}

Notes. See Sect. 2 for explanations.

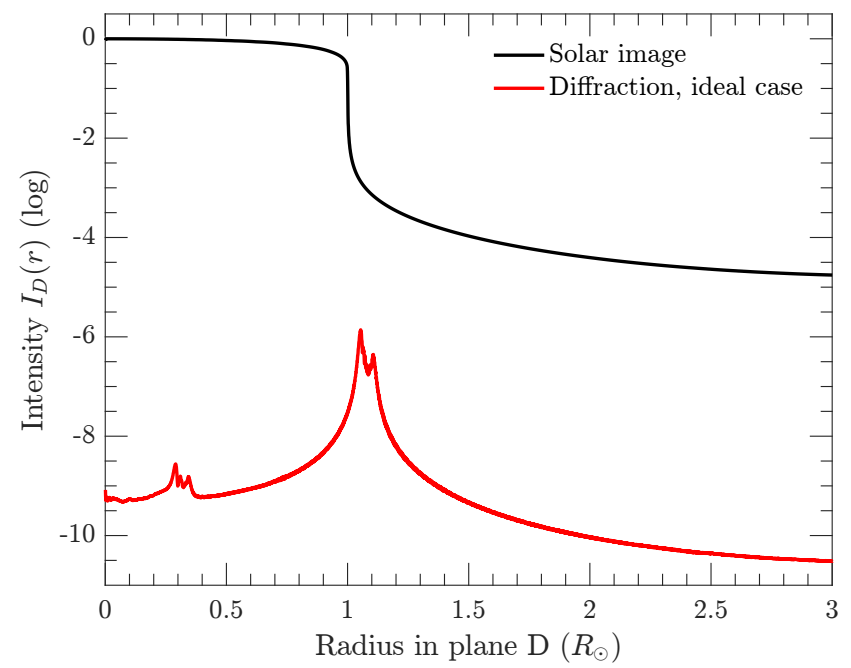

Fig. 2. Radial integrated intensity $I_{D}(r)$ in plane $D$, in logarithmic scale, in the ideal case without any perturbation, with the parameters of the study. The radius $r$ is given in units of $R_{\odot}$. The blackline shows the raw image of the solar disk - without occulting masks. The red line shows residual diffracted sunlight. From Rougeot (2017).

distribution function (BTDF). We note that we work here in transmission as we consider lenses, as opposed to the case using mirrors. The BTDF is commonly defined in a radiometric approach, as the ratio of the radiance (power per unit of solid angle per unit of area) of the transmitted scattered light, to the irradiance (power per unit of area) of the incoming light. It is a function of the scatter direction, counted from the specular direction, and it is expressed in $\mathrm{sr}^{-1}$. Additionally, the incoming irradiance can also be function of the incidence angle. We refer the reader to Mc Cluney (1994) for an extensive description of such function. Nevertheless, this strict radiometric formalism is not adapted to our wave propagation models. This section presents the model we developed to account for these effects.

For our study, we investigated only the scattering from the primary objective, that is, plane $A$ because it is known as the most critical for coronagraphy, as shown from past instruments (Lyot 1939; Brueckner et al. 1995). The reason is that this optical surface receives the most diffracted sunlight, after the external occulter. A similar analysis considering the other lenses - planes 
$O^{\prime}$ and $C$ - can easily be performed, following the exact same methodology.

\subsection{Theoretical considerations}

Our approach is based on Harvey surface scatter theory, presented in Harvey et al. (2007, 2009) for instance. In this section, we explain the methodology we developed, and the related numerical implementation using a discrete representation.

The surface roughness is modeled by a two dimension distribution function $h(x, y)$ which gives the algebraic height of any point $(x, y)$ with respect to the reference surface. The wave front perturbation $\mathcal{W}_{r}$ associated with the surface roughness is written as

$\mathcal{W}_{r}(x, y)=\exp \left(\frac{2 i \pi}{\lambda}(n-1) \times h(x, y)\right)$

where $n$ is the refractive index of the lens, fixed to $n=1.5$ here.

In most applications, the roughness structure is assumed isotropic and normally distributed, but is spatially correlated in a way depending on the manufacturing and polishing processes. The roughness $h(x, y)$ is thus computed as the convolution product of an uncorrelated white noise $h_{u c}(x, y)$ by a spatial filter $g(x, y)$. Alternatively, $h(x, y)$ can be computed by filtering in the frequency domain with the filter $G=\mathcal{F}[g]$ :

$$
\begin{aligned}
h(x, y) & =h_{u c}(x, y) \circledast g(x, y) \\
& =\mathcal{F}^{-1}\left[\mathcal{F}\left[h_{u c}\right]\left(f_{x}, f_{y}\right) \times G\left(f_{x}, f_{y}\right)\right](x, y)
\end{aligned}
$$

where $\left(f_{x}, f_{y}\right)$ are the spatial frequencies. In practice, the spatial spectrum of the roughness is band-limited (Harvey et al. 2012). For scattering, the range is $[1 / D ; 1 / \lambda]$, where $D$ is the characteristic dimension of the surface - the pupil diameter for instance. Lower frequencies are related to conventional optical aberrations, while frequencies above $1 / \lambda$ are irrelevant.

In order to simulate numerically a surface height that is consistent with a prescribed target roughness, we need to determine the correct filter impulse response $g(x, y)$, or equivalently its transfer function $G\left(f_{x}, f_{y}\right)$. In addition, special care must be taken because our model is based on a discrete representation. Considering a random surface structure $h(x, y)$ sampled on a regular grid of $N \times N$ points with sampling $s$, its power spectral density (PSD) $\mathcal{P}_{h}$ is defined as

$\mathcal{P}_{h}\left(f_{x}, f_{y}\right)=\frac{1}{N^{2}} \times\left\langle\left|\mathcal{F}[h]\left(f_{x}, f_{y}\right)\right|^{2}\right\rangle$

where $\langle\cdot\rangle$ denotes the expected value. The PSD of the filtered discrete process $h$ is related to that of the white noise input process $h_{u c}$ by

$\mathcal{P}_{h}\left(f_{x}, f_{y}\right)=\mathcal{P}_{h_{u c}}\left(f_{x}, f_{y}\right) \times\left|G\left(f_{x}, f_{y}\right)\right|^{2}$

where $\mathcal{P}_{h_{u c}}\left(f_{x}, f_{y}\right)=1$ is the PSD of a sampled white noise we note that Eq. (13) also applies to $h_{u c}$. As already stated, the roughness is assumed isotropic. The PSD is thus even and radial in the Fourier space, so $G$ is, by construction. The filter $g$ is thus necessarily real.

Our goal is to find $G$ in Eq. (14) so that the PSD of the sampled surface $h$ matches the PSD provided by a specific model denoted by $\mathcal{P}_{\text {mod }}\left(f_{x}, f_{y}\right)$. We note that Eq. (14) only constrains $|G|^{2}$, which means that solution for $\mathrm{G}$ is up to a complex phase factor. We are however not interested in describing the whole set of solutions, because we only need to find one filter which guarantees that the resulting PSD correspond to the targeted one, i.e., $P_{\text {mod. }}$.
In the literature, $\mathcal{P}_{\text {mod }}\left(f_{x}, f_{y}\right)$ is often provided in the form of the PSD of a continuous random process, instead of a sampled one. Because the PSD of a sampled process is equal to the PSD of the continuous process multiplied by the total bandwidth, i.e., $(1 / s)^{2}$ in our case, we write

$\mathcal{P}_{h}\left(f_{x}, f_{y}\right)=\frac{1}{s^{2}} \mathcal{P}_{\bmod }\left(f_{x}, f_{y}\right)$

and thus we obtain from Eq. (14)

$G\left(f_{x}, f_{y}\right)=\frac{\sqrt{\mathcal{P}_{\text {mod }}\left(f_{x}, f_{y}\right)}}{s}$.

We note that in this discrete representation, the sampling step in the Fourier space is given by $s_{f}=1 /(N s)$, and the highest frequency in $f_{x}$ and $f_{y}$ is $f_{l}= \pm 1 / 2 s$.

The root-mean-square (RMS) roughness $\sigma_{r}$, which is characteristic of the finishing of the surface, is defined by

$\sigma_{r}^{2}=\frac{1}{S} \times \iint_{S} h^{2}(x, y) \mathrm{d} x \mathrm{~d} y$

where $S$ is the considered area - the entrance aperture for instance. Numerically, this value can be obtained by replacing the integral by a discrete sum over the grid, by replacing $\mathrm{d} x \mathrm{~d} y$ by $s^{2}$ and $\frac{1}{S}$ by $1 /\left(N^{2} s^{2}\right)$, leading to the sample variance of $h$. We note that Eq. (17) provides a value of the roughness corresponding to one particular realization of $h$. The related theoretical value, of which Eq. (15) is an estimate, can be obtained from the Parseval-Plancherel theorem

$\sigma_{r}^{2}=\iint \mathcal{P}_{\text {mod }}\left(f_{x}, f_{y}\right) \mathrm{d} f_{x} \mathrm{~d} f_{y}$

where the integration is over the considered frequency band. This theoretical variance can be evaluated by replacing the integral by a discrete sum over all frequencies of the discrete grid, and by replacing $\mathrm{d} f_{x} \mathrm{~d} f_{y}$ by $1 /\left(N^{2} s^{2}\right)$.

We now consider an incoming planar wave with normal incidence onto the plane with a roughness $h$. The BTDF is the diffraction at infinity of this wave front by the rough surface. In other words, it consists of the angular spectrum of the perturbation $\mathcal{W}_{r}(x, y)$ (Goodman 2005), whose frequencies are given by $\sin \gamma_{x} / \lambda$ and $\sin \gamma_{y} / \lambda$, where $\gamma_{x}, \gamma_{y}$ denote the two scatter angles from the direction of propagation $z$, in the $x$ - and $y$-directions, respectively. Using the small angle approximation, we have $\sin \gamma_{x} \approx \gamma_{x}$ and $\sin \gamma_{y} \approx \gamma_{y}$. Moreover, since we assumed the roughness is isotropic, the BTDF is statistically axisymmetric in expectation, and we can reduce the problem to a single scatter angle $\gamma_{r}=\sqrt{\gamma_{x}^{2}+\gamma_{y}^{2}}$. The BTDF is thus written as

$\operatorname{BTDF}\left(\gamma_{r}\right)=\left\langle\frac{1}{\lambda^{2}}\left|\mathcal{F}_{\lambda}\left[\mathcal{W}_{r}\right]\right|^{2}\right\rangle$.

In fact, $h$ is very small in astronomical application (Harvey et al. 2007) - a fraction of $\lambda$, and the complex exponential in Eq. (19) can be expanded. Injecting this expansion in Eq. (17), and leaving apart the specular component term, we obtain

$$
\begin{aligned}
\operatorname{BTDF}\left(\gamma_{r}\right) & =\left\langle\frac{1}{\lambda^{2}}\left|\frac{2 i \pi}{\lambda}(n-1) \times \mathcal{F}_{\lambda}[h]\right|^{2}\right\rangle \\
& =\frac{4 \pi^{2}(n-1)^{2}}{\lambda^{4}} \times \mathcal{P}_{\text {mod }}\left(\frac{\gamma_{r}}{\lambda}\right) .
\end{aligned}
$$

Our Eq. (20) is consistent with Eq. (1) of Harvey et al. (2007) working in reflection instead of transmission replaces the factor $(n-1)$ with 2 . 


\subsection{Definition of the $P S D$ model $\mathcal{P}_{\text {mod }}$}

Based on studies of the nature of the micro-structure, Harvey et al. (2009) proposed a model for the PSD using an isotropic $\mathrm{ABC}$ function (or K-correlation) of the form

$\mathcal{P}_{\text {mod }}\left(f_{r}\right)=K \times \frac{A B}{\left(1+\left(B f_{r}\right)^{2}\right)^{\frac{C+1}{2}}}$

with $\quad K=\frac{1}{2 \sqrt{\pi}} \frac{\Gamma\left(\frac{C+1}{2}\right)}{\Gamma\left(\frac{C}{2}\right)}$ and $f_{r}=\sqrt{f_{x}^{2}+f_{y}^{2}}$

where $\Gamma(\cdot)$ is the usual Gamma function, and $A, B$ and $C$ the three parameters of the model. $A$ drives the amplitude of the PSD curve, or the "plateau", $B$ defines the "knee" and $C$ the slope. Such a PSD defines a low-pass filter $G$.

We chose the following formulation for our study. In order to estimate $A, B$, and $C$ for ASPIICS, our approach consisted in fitting the BTDF curve computed using Eq. (20) to a given curve of the primary objective L1, which was based on experimental measurements performed on a demonstration model of the lens. The derived parameters are $A=3.75 \times 10^{-1} \mathrm{~nm}^{2} \mathrm{~mm}$, $B=179 \mu \mathrm{m}$ and $C=0.82$. These values are of the same orders of magnitude as the ones described in Harvey et al. (2012). The RMS roughness is $\sigma_{r}=1.92 \mathrm{~nm}$ from Eq. (16), about $\lambda / 286$. This value is well in line with our expectations, in other words, a few nanometers.

\subsection{Numerical implementation}

The numerical implementation was done as follows. From a given PSD model $\mathcal{P}_{\text {mod }}$, we computed the filter $G$ from Eq. (16). We also computed a single realization of white noise $h_{u c}$ with a variance 1 on the sampling grid $N \times N$. The micro-structures $h(x, y)$ were then computed following Eq. (12), using standard two dimensional FFT routines in Matlab, and taking care of the normalization factor $1 / N$ due to the discrete representation. The negligible imaginary residuals were eliminated. Finally, the wave front error in plane $A$ is given by Eq. (11). For the sake of reproducible research, we provide the numerical code (Matlab/Octave) online, with the seed used for the simulation ${ }^{1}$.

The band-limitation $[1 / D ; 1 / \lambda]$ mentioned above must be discussed. To comply with the upper limit, we should theoretically consider a sampling in plane $A s_{A}=2 \lambda=1.1 \mu \mathrm{m}$, which is numerically very costly. For instance, this would require 45455 points only to pad the entrance aperture - without accounting for zero-padding. With our sampling of $18.5 \mu \mathrm{m}$ chosen for the study, we get $f_{l}= \pm 2.70 \times 10^{4} \mathrm{~m}^{-1}$, which gives a maximum scattered angle of $\gamma_{r}=1.71^{\circ}$. The total field-of-view of ASPIICS is $1.6^{\circ}$, i.e., $6 R_{\odot}$. Therefore, we considered the inherent bandlimitation of our model acceptable, since involved scatter angles are sufficient. Regarding the lower boundary, the first non-null frequency is $s_{f}=6.6 \times 10^{-1} \mathrm{~m}^{-1}$, which is thus lower than $1 / D$.

Finally, we remind the reader that the sampling $s_{A}$, and so the frequency domain, is function of the size of the arrays $N$, as given in Rougeot (2017). This is an inherent feature of our model, which is of importance to understand and reproduce the results reported in this paper. The domain the PSD is defined on is $\pm 1 /\left(2 s_{A}\right)$, and thus depends on $N$. Since $\sigma_{r}^{2}$ is the integral of the PSD in the frequency domain, changing the value of $N$ causes a change of the computed value of $\sigma_{r}$. This can yet be avoided by fixing the value of $s_{A}$ independently, in other words, not using

1 https://github.com/rrougeot/FourierOptics

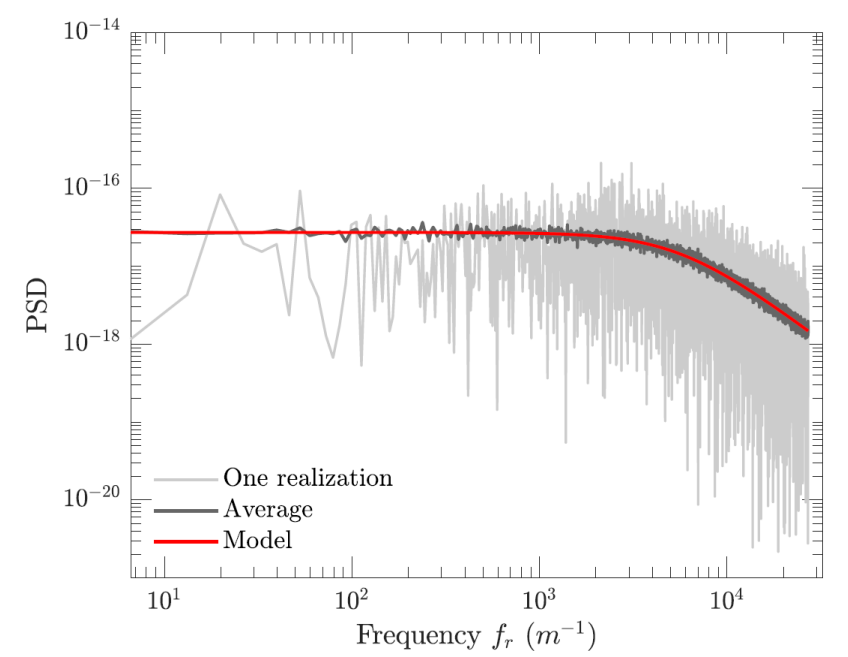

Fig. 3. Power spectral density of the simulated micro-roughness structure, as a function of spatial frequency $f_{r}$. The frequency domain is restricted to $f_{l}=1 / 2 s_{A}=2.70 \times 10^{4} \mathrm{~m}^{-1}-$ for $N=2^{13}$ and $s_{A}=$ $18.5 \mu \mathrm{m}$. The red line shows the model curve $\left(1 / s_{A}\right)^{2} \times \mathcal{P}_{\bmod }$, with our selected $A, B$, and $C$ parameters. Light gray: radial cut of one random realization $\mathcal{P}_{h}$. The dark gray line shows the average of 100 random realizations.

the mentioned approach so that the domain of the PSD no longer depends on the size of the array $N$ used in the simulation. We note that the value $N=2^{13}$ was determined in Rougeot (2017) as a compromise between computational time and precision, governed by aliasing. Here, we chose to keep this value, even if the aliasing is expected to have a bigger impact because of the scattering. If one seeks for a much accurate computation, a larger value of $N$ is preferable. We compared our results with a computation using $N=2^{15}$, and the relative difference is less than $10 \%$ in the useful field-of-view of ASPIICS. Moreover, our curves are smooth, while this would not be the case with $N=2^{12}$.

In Fig. 3, we plot the PSD model $\mathcal{P}_{\text {mod }}$, and we superimposed a radial cut of the single random realization of the PSD $\mathcal{P}_{h}\left(f_{x}, f_{y}\right)$, computed by Eq. (14), and a statistical average of 100 other random realizations using different seeds, to simulate Eq. (13). As observed, the curves coincide well, and the expectation correctly converges to the theoretical model.

In Fig. 4, we show the micro-structures $h(x, y)$ over an area of $4 \mathrm{~mm}$ by $4 \mathrm{~mm}$. The spatial correlation is clearly visible, with a characteristic length $\simeq 150 \mu \mathrm{m}$, which is consistent with the value of $B$.

Finally, we illustrate in Fig. 5 the speckles induced by the wave front error. Here, we enhanced the observed effect by taking $100 \times A$ instead of $A$, for sake of qualitative illustration. The related RMS roughness is $\sigma_{r}=21 \mathrm{~nm}$, which is very high with respect to the typical value used in the application described in this paper. Figure 5 plots a radial cut of the PSF of the on-axis point source in the focal plane of the telescope, that is, plane $B$. We superimposed the ideal PSF, for direct comparison. We show the corresponding two dimension images in Fig. A.1.

\section{Effects of scattering}

\subsection{Analysis of the intensity}

To provide a complete understanding of the effects of scattering, we analyzed the intensities in the successive planes $O^{\prime}, C$, and $D$ of the coronagraph, and compared them to the reference case without scattering. 


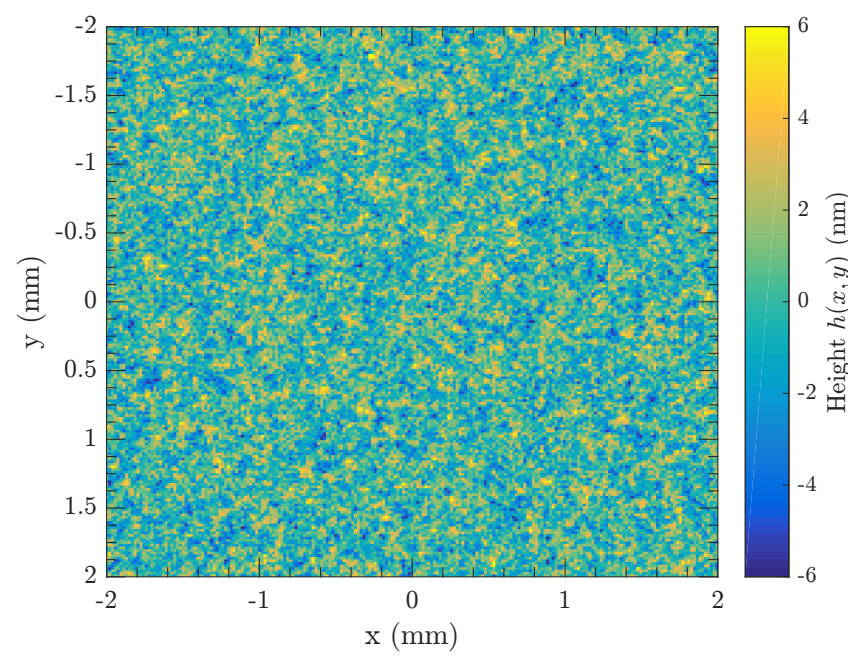

Fig. 4. Height $h(x, y)$ of the micro-structures in plane $A$, in nanometers. The area is $4 \mathrm{~mm}$ by $4 \mathrm{~mm}$. The spatial sampling is $s_{A}=18.5 \mu \mathrm{m}$

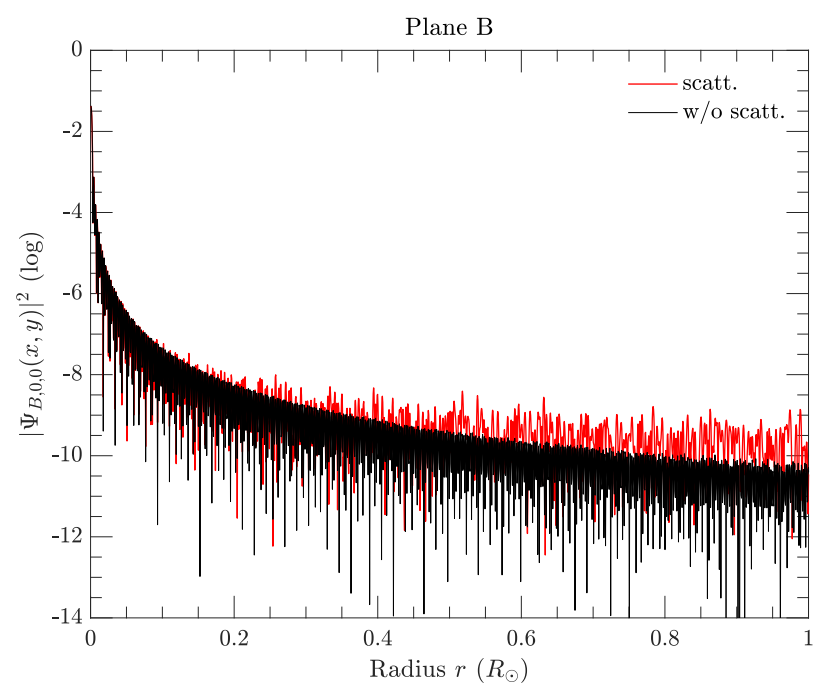

Fig. 5. Radial cut of the PSF $\left|\Psi_{B, 0,0}(r)\right|^{2}$ in plane $B$, in logarithmic scale. The radius $r$ is given in units of $R_{\odot}$. In red we show the PSF with speckles, due to scattering in plane $A\left(\sigma_{r}=21 \mathrm{~nm}\right)$. In black we show the ideal PSF.

In Fig. 6 panel a, we plot in red the radial intensity $I_{O^{\prime}}(r)$ in plane $O^{\prime}$ in logarithmic scale. The diffraction appears as a narrow fringe located at the position of the image of the external occulter edge, i.e., $1.057 R_{\odot}$, as explained in Fig. 7 of Rougeot (2017). The reference curve of the ideal case without scattering is also given in black, for direct comparison. As first, no noticeable difference is observed between the two curves. Panel $b$ gives the relative difference, in logarithmic scale. The color of the dots indicate the sign of the relative difference - see the caption of Fig. 6. When introducing the wave front error $\mathcal{W}_{r}$ in plane $A$, the intensity $I_{O^{\prime}}(r)$ around the diffraction peak $1.057 \pm 0.005 R_{\odot}$ is reduced by $\simeq 0.01 \%$, compared to the ideal case. Even if this loss is very small in percentage, the high dynamic range of the diffraction intensity makes the impact more noticeable in the rest of the field-of-view - the peak intensity is higher by several orders of magnitude. Accordingly, the intensity outside the peak increases from $1 \%$ at $1.2 R_{\odot}$ to almost $20 \%$ at $3 R_{\odot}$, with an average of $12 \%$. We checked that the total integrated intensity over the complete plane $O^{\prime}$ is identical in both cases without and with scattering.

The effect of the perturbations from surface roughness in plane $A$ is to spread the diffraction intensity $I_{O^{\prime}}$ over the plane $O^{\prime}$. The diffraction peak becomes slightly less bright. The direct consequence is that the internal occulter cuts out less diffraction. To quantify that, we looked the integrated flux that is not blocked by the occulter

$\Phi_{O^{\prime}}=\int_{0}^{2 \pi} \int_{0}^{3 R_{\odot}} I_{O^{\prime}}(r) \times \mathcal{M}(r) r \mathrm{~d} r \mathrm{~d} \theta$.

The interesting value is in fact the ratio of $\Phi_{O^{\prime}}$ between the case with scattering and without. This ratio is 1.0071 , which means that $0.71 \%$ more diffraction flux will leak past the occulter.

Figure 7 presents the same results for plane $C$. There, the diffraction is a peak located at the geometrical image of the pupil radius (Rougeot 2017). As shown in panel a, the intensity $I_{C}(r)$ has increased in the central region of the field $r<R_{\mathrm{p}}$, with respect to the ideal case. The relative difference given in panel $b$ shows that it is almost the double - a relative difference of almost $100 \%$. Because the Lyot stop does not filter out this central region, a much bigger flux of diffracted light will thus reach plane $D$. At last, we verified that the observed difference of the total integrated intensity over the whole plane $C$ correctly matches to the observation made from plane $O^{\prime}$.

Finally, in Fig. 8 we plots the radial intensity $I_{D}(r)$ in plane $D$, in logarithmic scale, with the relative difference as well. Whereas the peak around $1.05 R_{\odot}$ is not impacted much, the diffraction at $3 R_{\odot}$ has increased by more than one order of magnitude with respect to the ideal case. The degradation of the performance is therefore significant.

\subsection{Sizing the internal occulter and the Lyot stop}

Based on the observation of the effects of scattering on the intensities in planes $O^{\prime}$ and $C$, we can intuit that sizing the internal occulter and the Lyot stop will not mitigate the degradation of the image much. We ran additional simulations using internal occulters of $1.729 \mathrm{~mm}(+100 \mu \mathrm{m}$ from $R$ image $)$ and $1.759 \mathrm{~mm}$ radius ( $+150 \mu \mathrm{m}$ from $R$ image), and Lyot stops of $95 \%$ and $93 \%$ the size of the pupil image, in order to verify this assumption.

In Figs. 9 and 10 we plot the radial intensity $I_{D}(r)$ in plane $D$ when sizing respectively the internal occulter and the Lyot stop. These results are consistent with the study of Rougeot (2017), where it has been shown that increasing the internal occulter reduces both the diffraction peak and diffraction tail, shifts the peak further in the field-of-view, and that reducing the Lyot stop mostly acts on the diffraction tail. However, in the case with the scattering, the effects on the diffraction tail $\geq 1.5 R_{\odot}$ is no longer present. Sizing the Lyot stop becomes thus ineffective; sizing the internal occulter remains relevant only for the inner field-of-view close to the solar limb.

This observed result is explained by the following reasoning, and is similar to the study of speckles made by Aime \& Soummer (2004) in the case of the classical Lyot coronagraph. In principle, in a coronagraph, the internal occulter acts as a high-pass filter, as it cuts out the light in the central region of the focal plane. In a similar way, the Lyot stop acts as low-pass filter. Their combined effects provide the rejection of the coronagraph. However, this is only effective on perfect planar wave fronts. The introduction of perturbations in the wave front deteriorate the rejection. The reasoning is as follows. 

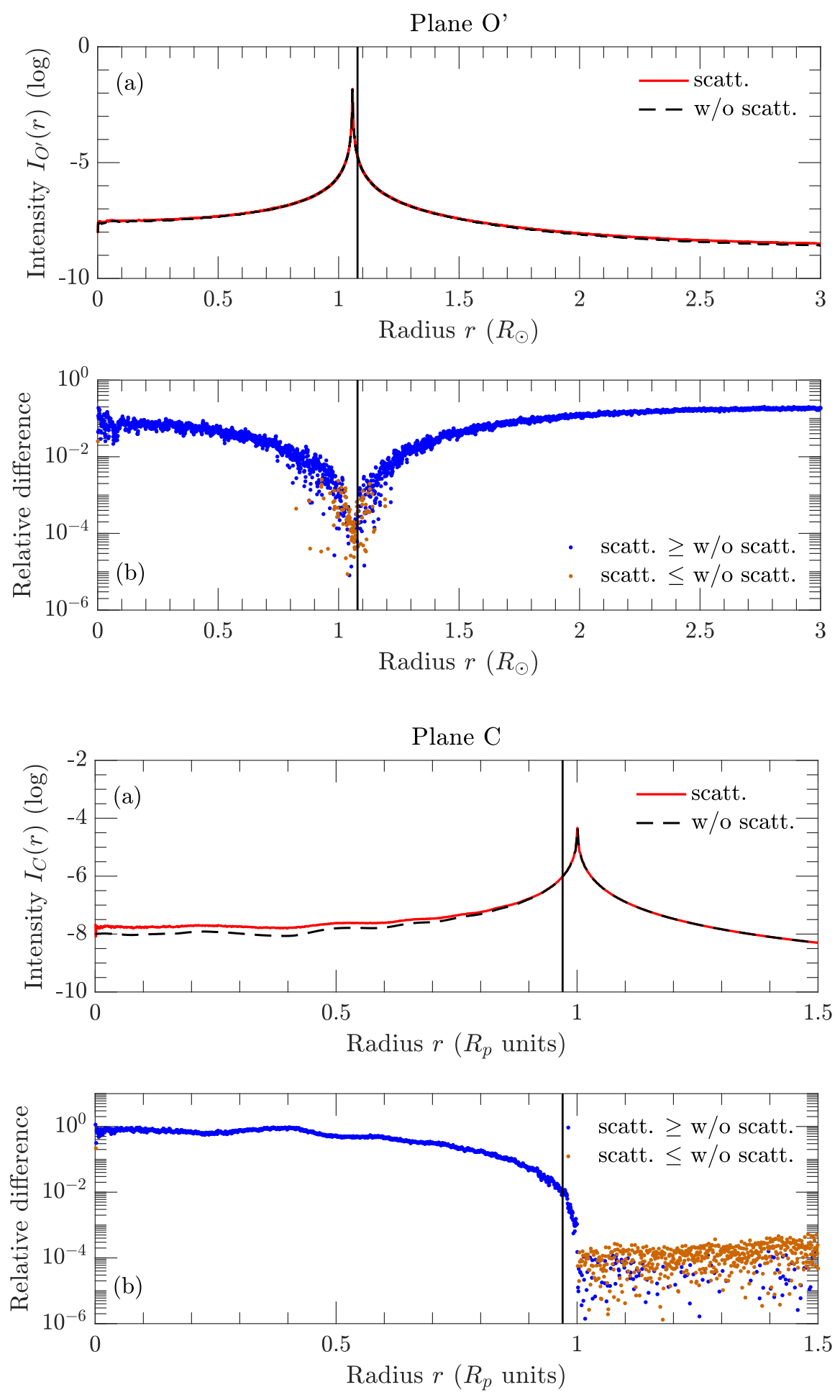

Fig. 6. Panel $a$ : radial intensity $I_{O^{\prime}}(r)$ in plane $O^{\prime}$, in logarithmic scale. The radius $r$ is given in units of $R_{\odot}$. In red we show the case with scattering in plane $A$. In black, the ideal case without scattering. The vertical line represents the internal occulter outer radius. Panel $b$ : relative difference, in absolute value. Blue dots indicate where the intensity $I_{O^{\prime}}(r)$ is higher in the case with scattering, and yellow dots indicate the contrary.

Fig. 7. Panel $a$ : radial observed intensity $I_{C}(r)$ in plane $C$, in logarithmic scale. The radius $r$ is given in units of $R_{\mathrm{p}}$. In red we plot case with scattering in plane $A$. Black shows the ideal case without scattering. The vertical line represents the Lyot stop radius. Panel $b$ : relative difference, in absolute value. Blue dots indicate where the intensity $I_{C}(r)$ is higher in the case with scattering, and yellow dots indicate the contrary.

Based on Eq. (6), the wave front in plane $C$ is of the form, with simplified notations

$\Psi_{C} \propto \mathcal{F}\left[\Psi_{O^{\prime}} \times \mathcal{M}\right] \times \varphi_{+z_{0}}$

$\Psi_{C} \propto \mathcal{F}\left[\mathcal{F}\left[\Psi_{A} \times \mathcal{P} \times \varphi_{-z_{0}} \times \mathcal{W}_{r}\right] \times \mathcal{M}\right] \times \varphi_{+z_{0}}$

$\Psi_{C} \propto\left(\widetilde{\Psi}_{A} \times \mathcal{W}_{r}-\left(\widetilde{\Psi}_{A} \times \mathcal{W}_{r}\right) \circledast \mathcal{F}[\overline{\mathcal{M}}]\right) \times \varphi_{+z_{0}}$

with $\quad \widetilde{\Psi}_{A}=\Psi_{A} \times \mathcal{P} \times \varphi_{-z_{0}}$ and $\overline{\mathcal{M}}=1-\mathcal{M}$

$\overline{\mathcal{M}}$ is the negative of the internal occulter mask.
In the ideal coronagraph, the wave front error is null so $\mathcal{W}_{r}=1$ in Eq. (25). The principle of the coronagraph consists of minimizing the term

$\Upsilon=\widetilde{\Psi}_{A}-\widetilde{\Psi}_{A} \circledast \mathcal{F}[\overline{\mathcal{M}}]$

inside the Lyot stop opening. In other words, as the Lyot stop acts as low-pass filter, the coronagraphic rejection is obtained when $\widetilde{\Psi}_{A}$ and $\widetilde{\Psi}_{A} \circledast \mathcal{F}[\overline{\mathcal{M}}]$ are similar in the low frequency domain (Ferrari et al. 2010) - the high frequencies are filtered out anyway. 
Plane D
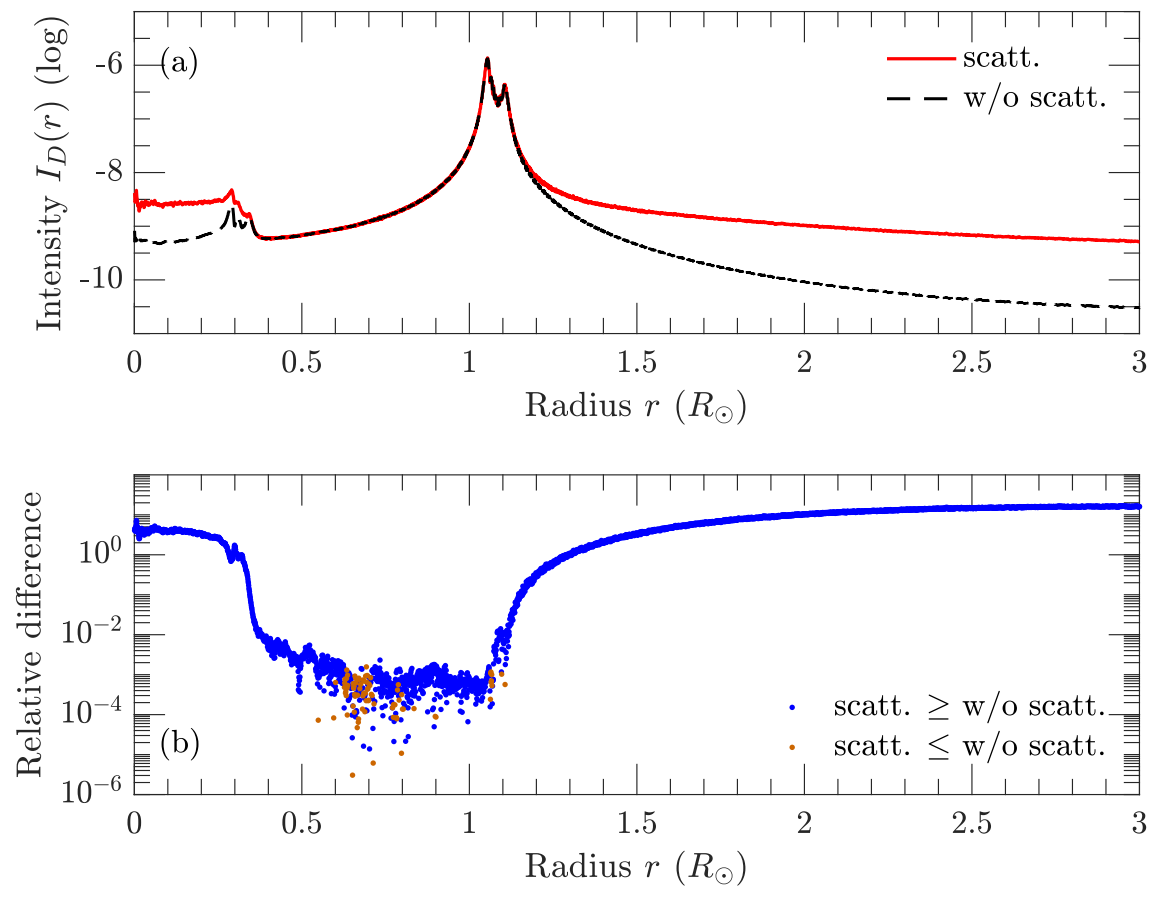

Fig. 8. Panel $a$ : radial intensity $I_{D}(r)$ in plane $D$, in logarithmic scale. The radius $r$ is given in units of $R_{\odot}$. In red we show the case with scattering in plane $A$. Black shows the ideal case without scattering. Panel $b$ : relative difference, in absolute value. Blue dots indicate where the intensity $I_{D}(r)$ is higher in the case with scattering, and yellow dots indicate the contrary.
We now consider the wave front error $\mathcal{W}_{r}$. The term given in Eq. (26) is no longer correct. As we did in Eq. (20), $\mathcal{W}_{r}$ can be expanded as

$W_{r} \simeq 1+i w_{r}$

and the term in Eq. (26) becomes

$\Upsilon^{\prime}=\Upsilon+i w_{r} \times \widetilde{\Psi}_{A}-\left(i w_{r} \times \widetilde{\Psi}_{A}\right) \circledast \mathcal{F}[\overline{\mathcal{M}}]$

While the term $\Upsilon$ is minimized inside the Lyot stop opening, as explained above, it is most likely not the case for the second term including $w_{r}$. Because of the random nature of $w_{r}$, the difference between the term $w_{r} \times \widetilde{\Psi}_{A}$ and its convolution with $\mathcal{F}[\overline{\mathcal{M}}]$ is relatively big, in the entire field and in particular inside the Lyot stop. As a result, a substantial diffraction energy is not cut-off, and propagates further to plane $D$.

This last result is consistent with Sect. 4 of Aime \& Soummer (2004), even if these authors considered ground-based coronagraph coupled with adaptive optics. While the coronagraph efficiently reduces the diffraction intensity produced by the coherent part of the wave, it does not act on the contribution from the random defects which perturb the coherency of the wave - that is, the speckles.

The main mitigation to control the scattering consists in fact of the polishing of the optics surface, to achieve the best roughness possible. Nevertheless, it depends on several other factors, such as the type of glass, or the polishing process.

\subsection{Sensitivity analysis on the PSD parameters}

Finally, we investigated the influence of each of the three components of the PSD model $\mathcal{P}_{\text {mod }}$. We varied independently their values by $25 \%, 50 \%, 150 \%$ and $200 \%$ with respect to the defined case ASPIICS. Table 2 reports the different study cases. Cases A1-A4 increase the amplitude $A$ of the PSD. Cases B1$\mathrm{B} 4$ look at a variation of the location of the shoulder. Because $B$

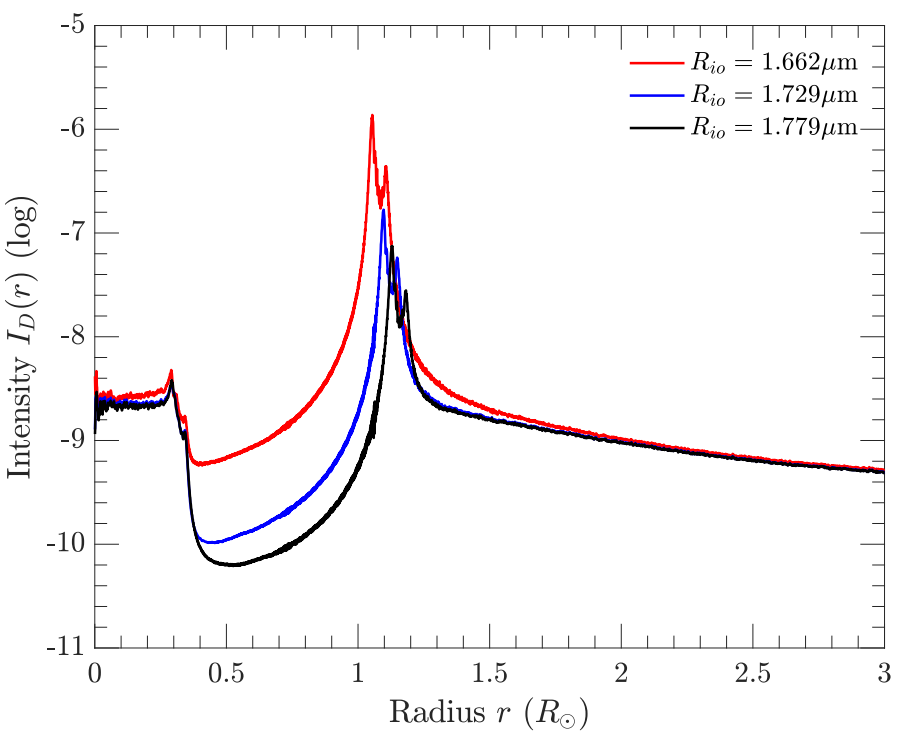

Fig. 9. Radial intensity $I_{D}(r)$ in plane $D$, in logarithmic scale, for different sizes of the internal occulter. The radius $r$ is given in units of $R_{\odot}$. The red line shows an internal occulter of $1.662 \mathrm{~mm}$, the blue line an internal occulter of 1.729 , and the black line an internal occulter of $1.779 \mathrm{~mm}$.

also contributes to the amplitude of $\mathcal{P}_{\text {mod }}$, see Eq. (21), we modified the value of $A$ accordingly in order to keep the amplitude of the PSD unchanged. Finally, cases $\mathrm{C} 1-\mathrm{C} 4$ vary the slope. Here again, the value of $A$ was tuned accordingly to compensate the change of value of $K$. All these cases give a RMS roughness $\sigma_{r}$ of $1-2 \mathrm{~nm}$.

Results are given in Figs. 11-13, for cases A1-A4, B1-B4, and $\mathrm{C} 1-\mathrm{C} 4$, respectively. In the figures, the left panel plots the PSD $\mathcal{P}_{\text {mod }}$, computed by Eq. (21). The frequency domain is limited to $f_{l}=1 / 2 s_{A}=2.70 \times 10^{4} \mathrm{~m}^{-1}$. The right panel plots the radial observed intensities $I_{D}(r)$ in plane $D$, in logarithmic 


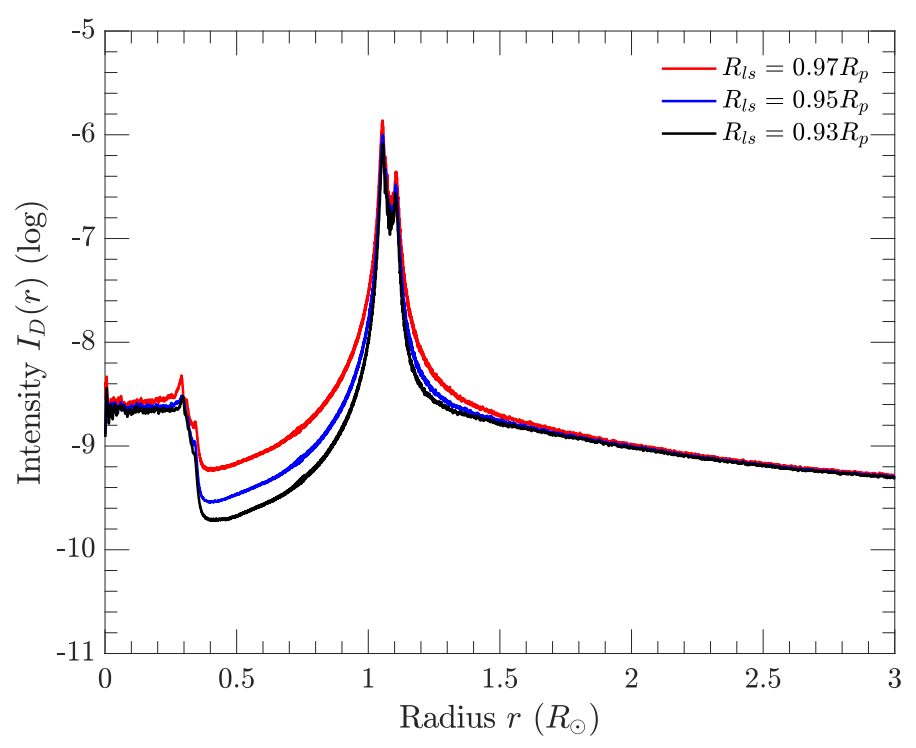

Fig. 10. Radial intensity $I_{D}(r)$ in plane $D$, in logarithmic scale, for different sizes of the Lyot stop. The radius $r$ is given in units of $R_{\odot}$. In red we show a Lyot stop of $0.97 R_{\mathrm{p}}$. In blue a Lyot stop of $0.95 R_{\mathrm{p}}$, and in black a Lyot stop of $0.93 R_{\mathrm{p}}$.

scale. We also superimposed as a black curve the case ASPIICS with the nominal parameters $A, B$, and $C$, and as a gray curve the ideal reference without scattering, for direct comparison. As expected, the residual diffracted sunlight increases in the range $1.1 R_{\odot}-3 R_{\odot}$ when the amplitude of the PSD increases (from case A1 to A4), when the shoulder is located further in the frequency domain (from case B4 to B1), and when the slope gets less steep (from case $\mathrm{C} 4$ to $\mathrm{C} 1$ ).

Even if the variation of $A$ acts on every frequency, the biggest impact is observed for the cases $B$ - for the same relative variations of the parameter. The total support of the PSD, and so the total scatter energy, varies much more from case $B 1$ to $B 4$. This is consistent with the corresponding values of $\sigma_{r}$ - see Table 2. Therefore, modifying the shoulder impacts the scattering the most, in comparison to the amplitude and the slope. We note that the PSD plots in Figs. 11-13 are in log-log scale, which may be misleading.

As a final analysis, we investigated the effect of the actual shape of the PSD on the diffraction intensity. We defined two study cases with the following set of parameters: $A=9.91 \times$ $10^{-1} \mathrm{~nm}^{2} \mathrm{~mm}, B=537 \mu \mathrm{m}$ and $C=1.5$ for case $\Sigma 1$, and $A=2.99 \times 10^{-1} \mathrm{~nm}^{2} \mathrm{~mm}, B=71.6 \mu \mathrm{m}$ and $C=1.0$ for case $\Sigma 2$ - recalled in Table 2 . For both, the values of $B$ and $C$ were fixed arbitrarily, then the value of $A$ was derived using a proportionality law so that the associated $\sigma_{r}$ is equal to the one of the case ASPIICS. Doing so, the actual shape of the PSD is different, but the RMS roughness remains identical. We note that related BTDF curves do not match to any real experimental data for these cases.

In the left panel of Fig. 14 we plot the PSD $\mathcal{P}_{\text {mod }}$ of cases $\Sigma 1$ and $\Sigma 2$, together with the one of case ASPIICS. As shown, all three curves are obviously of different shape. The figure plots in the right panel the radial observed intensities $I_{D}(r)$ in plane $D$ of the cases, in logarithmic scale, and gives the ideal reference without scattering as gray curve.

In the case $\Sigma 1$, the diffraction intensity is bigger in the inner region $1.1 R_{\odot}-1.5 R_{\odot}$ compared to the case ASPIICS, but lower in the outer field-of-view. For the case $\Sigma 2$, it is the contrary: the
Table 2. Study cases for the influence of $A, B$, and $C$ parameters of the Harvey model of the PSD $\mathcal{P}_{\text {mod }}$.

\begin{tabular}{lcccc}
\hline \hline Case & $A\left(\mathrm{~nm}^{2} \mathrm{~mm}\right)$ & $B(\mu \mathrm{m})$ & $C$ & $\sigma_{r}(\mathrm{~nm})$ \\
\hline ASPIICS & $3.75 \times 10^{-1}$ & 179 & 0.82 & $1.92(\lambda / 286)$ \\
\hline A1 & $9.38 \times 10^{-2}$ & 179 & 0.82 & $0.953(\lambda / 577)$ \\
A2 & $1.875 \times 10^{-1}$ & 179 & 0.82 & $1.35(\lambda / 408)$ \\
A3 & $5.625 \times 10^{-1}$ & 179 & 0.82 & $2.35(\lambda / 235)$ \\
A4 & $7.50 \times 10^{-1}$ & 179 & 0.82 & $2.70(\lambda / 204)$ \\
\hline B1 & 1.50 & 44.7 & 0.82 & $4.00(\lambda / 138)$ \\
B2 & $7.50 \times 10^{-1}$ & 89.5 & 0.82 & $2.92(\lambda / 188)$ \\
B3 & $2.50 \times 10^{-1}$ & 269 & 0.82 & $1.44(\lambda / 382)$ \\
B4 & $1.875 \times 10^{-1}$ & 358 & 0.82 & $1.16(\lambda / 474)$ \\
\hline C1 & 1.149 & 179 & 0.205 & $2.57(\lambda / 214)$ \\
C2 & $6.375 \times 10^{-1}$ & 179 & 0.41 & $2.32(\lambda / 237)$ \\
C3 & $2.832 \times 10^{-1}$ & 179 & 1.23 & $1.60(\lambda / 344)$ \\
C4 & $2.359 \times 10^{-1}$ & 179 & 1.64 & $1.37(\lambda / 401)$ \\
\hline$\Sigma 1$ & $9.91 \times 10^{-1}$ & 537 & 1.5 & $1.92(\lambda / 286)$ \\
$\Sigma 2$ & $2.99 \times 10^{-1}$ & 71.6 & 1.0 & $1.92(\lambda / 286)$ \\
\hline
\end{tabular}

Notes. Related RMS roughness $\sigma_{r}$ is also given, computed for $s_{A}=$ $18.5 \mu \mathrm{m}$.

intensity is lower in the region $1.1 R_{\odot}-1.8 R_{\odot}$, but bigger in the outer field-of-view.

The explanation of this observation is the following. As we presented, the scattering behavior is described by the BTDF, which gives the scattered power as a function of the scatter angle. This function is related to the PSD model as written in Eq. (20). The more power contained at low frequencies in the PSD, the more scattering at low scatter angles. Looking at the shapes of the PSD in Fig. 14 (left panel), we deduce that the scattering behavior is predominant at low scatter angle for case $\Sigma 1$, and at larger scatter angle for case $\Sigma 2$, with respect to the case ASPIICS. This is what we observe in the spatial distribution of the diffraction intensity in the right panel of Fig. 14. The scattered intensity is brighter close to the diffraction peak for case $\Sigma 1$, and brighter at large solar radius for case $\Sigma 2$.

\section{Conclusion}

In this paper, we investigated the influence of surface roughness of the telescope on the diffracted residual sunlight for the externally occulted Lyot solar coronagraph. The roughness is controlled by three parameters and is in the order of the nanometer (RMS). While the diffraction peak intensity observed on the detector at $\simeq 1.1 R_{\odot}$ is not impacted, the scattered diffraction tail significantly increases, up to one order of magnitude at large solar radius. The degradation of performance in the outer fieldof-view of the coronagraph is thus noticeable. We show that the actual shape of the power spectral density of the surface roughness drives the scattering behavior, and how the spatial distribution of diffraction intensity across the field-of-view is impacted.

We can compare these observed effects to the ones caused by a misalignment or off-pointing of the externally occulted solar coronagraph, as studied by Shestov \& Zhukov (2018). These authors show that the residual diffraction intensity increases evenly in the entire field-of-view for such effects, and not only at large $R_{\odot}$ as we observed for scattering. This is the first difference. The second difference is the level of degradation: Shestov \& Zhukov (2018) computed an increase of about $15 \%$ for a typical shift of the Sun of $5 \mathrm{arcsec}$, which corresponds to a lateral displacement of the pupil from the umbra center of about 
R. Rougeot et al.: Influence of surface roughness on diffraction in the externally occulted Lyot solar coronagraph
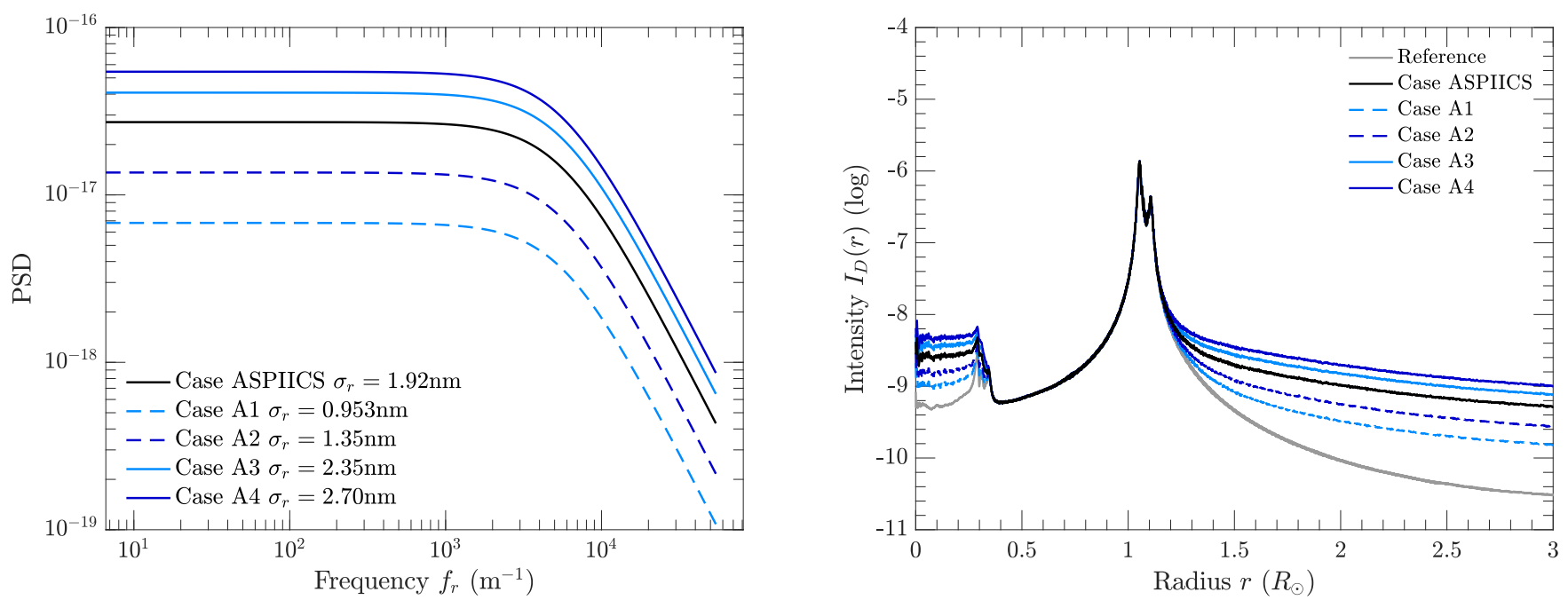

Fig. 11. Study cases A1-A4 - see Table 2. Left panel: power spectral density $\mathcal{P}_{\text {mod }}\left(f_{r}\right)$, in logarithmic scale. Right panel: radial intensity $I_{D}(r)$ in plane $D$, in logarithmic scale. The black curve represents the case ASPIICS. The gray curve represents the ideal reference without scattering.
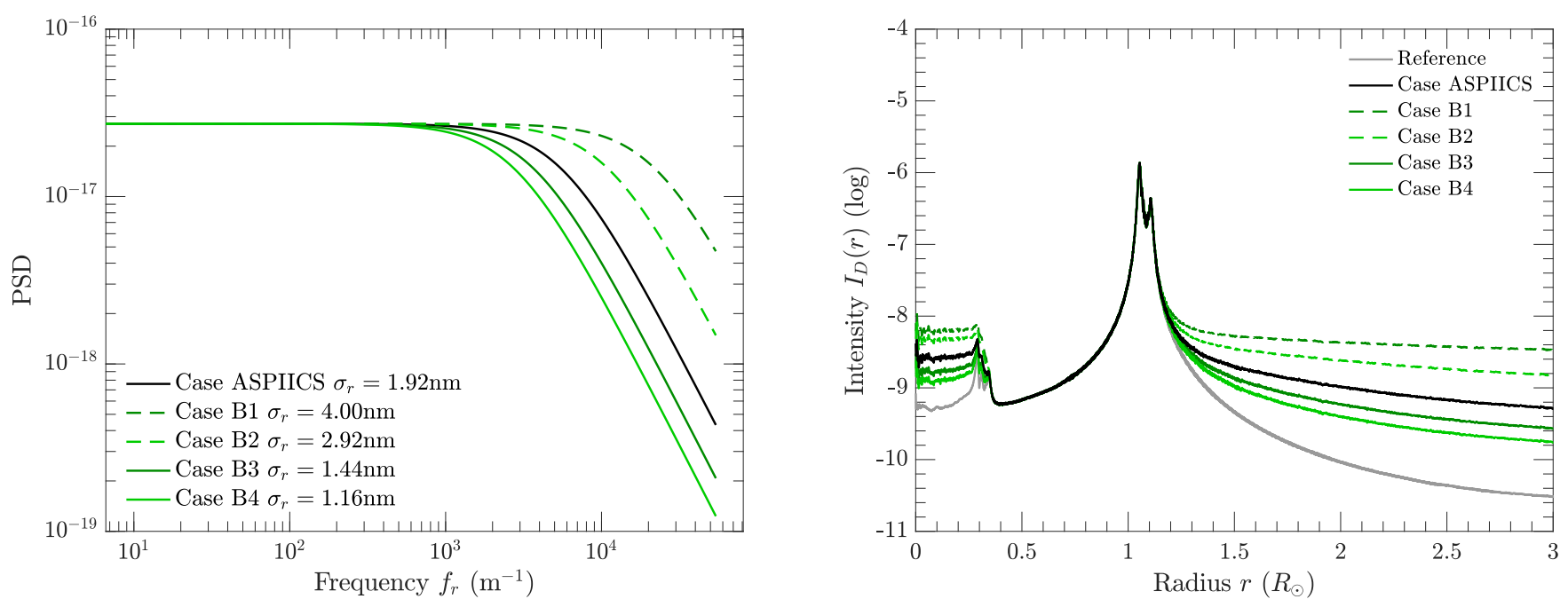

Fig. 12. Study cases A1-A4 - see Table 2. Left panel: power spectral density $\mathcal{P}_{\text {mod }}\left(f_{r}\right)$, in logarithmic scale. Right panel: radial intensity $I_{D}(r)$ in plane $D$, in logarithmic scale. The black curve represents the case ASPIICS. The gray curve represents the ideal reference without scattering.
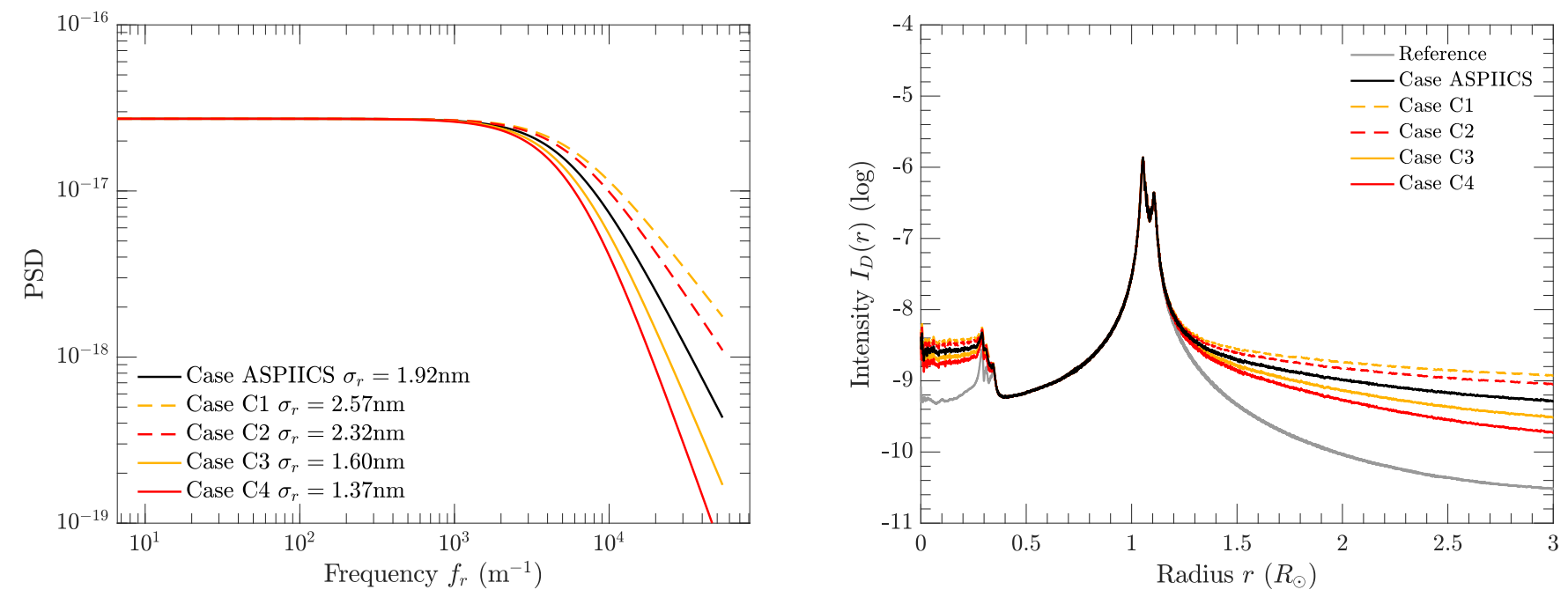

Fig. 13. Study cases A1-A4 - see Table 2. Left panel: power spectral density $\mathcal{P}_{\text {mod }}\left(f_{r}\right)$, in logarithmic scale. Right panel: radial intensity $I_{D}(r)$ in plane $D$, in logarithmic scale. The black curve represents the case ASPIICS. The gray curve represents the ideal reference without scattering. 

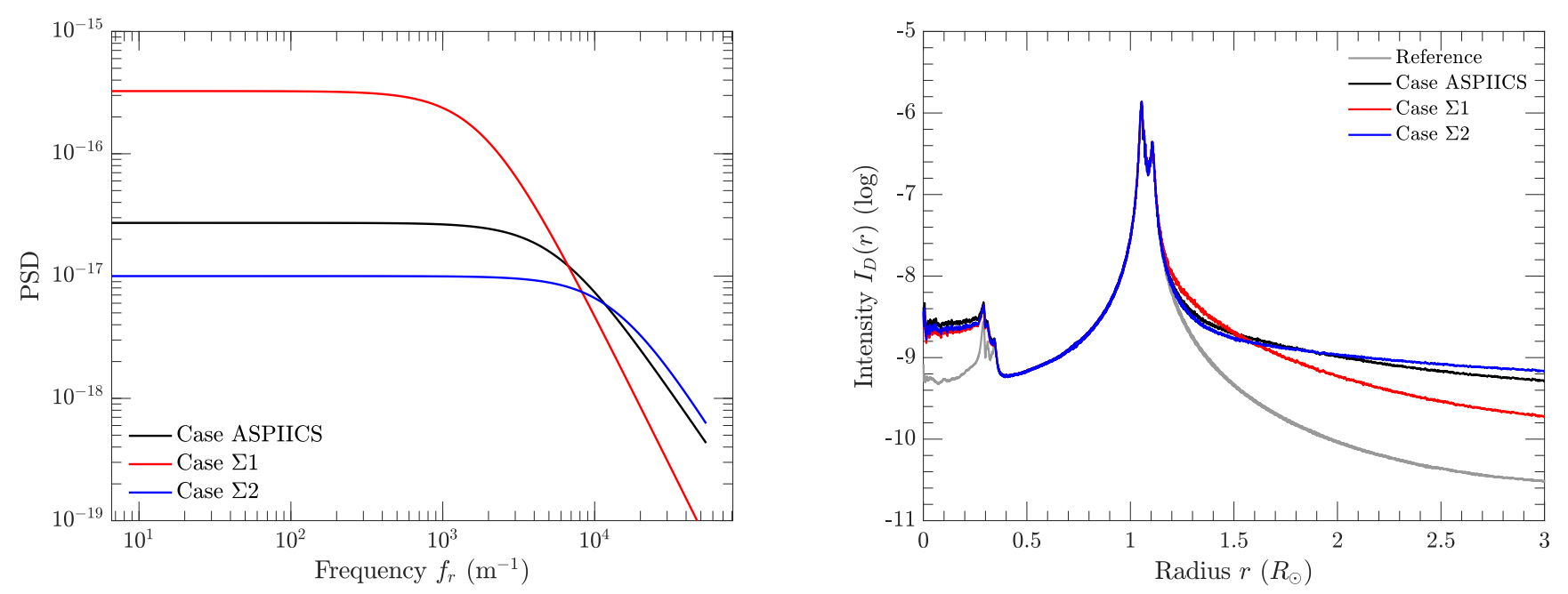

Fig. 14. Study cases $\Sigma 1$ and $\Sigma 2$ - see Table 2. Left panel: power spectral density $\mathcal{P}_{\bmod }\left(f_{r}\right)$, in logarithmic scale. Right panel: radial intensity $I_{D}(r)$ in plane $D$, in logarithmic scale. The black curve represents the case ASPIICS. The gray curve represents the ideal reference without scattering.

$3.5 \mathrm{~mm}$ at $144 \mathrm{~m}$ from the occulting disk - see their Fig. 8. In case of a typical off-pointing of the coronagraph by 10 arcsec, the computed increase is about three times: their Fig. 9. Considering the typical values for ASPIICS, the influence of surface roughness on performance is thus much more stringent, as we found an increase of the diffraction of up to one order of magnitude.

Finally, we also show that sizing the internal occulter or the Lyot stop does not provide any substantial effects on the scattering. The coronagraph acts indeed efficiently on perfect wave fronts, and any defects, seen as perturbation of the light coherency, will degrade the desired rejection effect. This result is consistent with that of Aime \& Soummer (2004).

Besides the results, we provide a methodology to implement the roughness scattering in the wave propagation model of Rougeot (2017). Similar analysis can be performed on the second and third optics, but these optics are known to be less critical for what concerns scattering in the final focal plane. This work is part of the overall end-to-end performance analysis for the ASPIICS solar coronagraph. Future work is foreseen to investigate the influence of the edge manufacturing tolerances of the external occulter.

Acknowledgements. We would like to thank D. Galano, C. Galy, V. Kirschner, M. N'Diaye, and J. Versluys for their help in this study. We also thank the European Space Agency and Thales Alenia Space for supporting this activity.

\section{References}

Aime, C. 2013, A\&A, 558, A138

Aime, C., \& Soummer, R. 2004, ApJ, 612, L85

Aime, C., Soummer, R., \& Ferrari, A. 2002, A\&A, 389, 334

Born, M., \& Wolf, E. 2006, Principles of Optics, 7th edn. (Cambridge: Cambridge University Press), 484
Bout, M., Lamy, P., Maucherat, A., Colin, C., \& Llebaria, A. 2000, Appl. Opt., 39, 22

Brueckner, G. E., Howard, R. A., Koomen, M. J., et al. 1995, Solar Phys., 162, 357

Cady, E. 2012, Opt. Express, 20, 15196

Cash, W. 2006, Nature, 442, 51

Cox, A. N. 2000, Allen's Astrophysical Quantities (Berlin: Springer)

Evans, J. W. 1948, Space Sci. Rev., 47, 95

Ferrari, A., Aime, C., \& Soummer, R. 2010, ApJ, 708, 218

Galano, D., Bemporad, A., Buckley, S., et al. 2018, Proc. SPIE, 10698, 106982 Y

Galy, C., Fineschi, S., Galano, D., et al. 2015, Proc. SPIE, 9604, 96040B

Goodman, J. W. 2005, Introduction to Fourier Optics (Greenwood Village, CO: Roberts and Company Publishers)

Harvey, J. E., Krywonos, A., \& Stover, J. C. 2007, Proc. SPIE, 6672, 66720C

Harvey, J. E., Choi, N., Krywonos, A., \& Marcen, J. G. 2009, Proc. SPIE, 7426, $74260 \mathrm{I}$

Harvey, J. E., Choi, N., Schroeder, S., \& Duparré, A. 2012, Opt. Eng., 51, 013402 Koutchmy, S. 1988, Space Sci. Rev., 47, 95

Lamy, P., Damé, L., Vivès, S., \& Zhukov, A. 2010, Proc. SPIE, 7731, 18

Landini, F., Mazzoli, A., Venet, M., et al. 2010, SPIE, 7735, 77354D

Landini, F., Romoli, M., Baccani, C., et al. 2016, Opt. Lett., 41, 757

Lenskii, A. V. 1981, AZh, 58, 648

Loreggia, D. 2015, Proc. SPIE, 9604, 96040F

Lyot, B. 1939, MNRAS, 99, 580

Mc Cluney, R. 1994, Introduction to Radiometry and Photometry (Norwood, MA: Artech House)

Newkirk, Jr., G., \& Bohlin, D. 1965, Ann. Astrophys., 28, 234

Peter, H., Bingert, S., Klimchuk, J. A., et al. 2013, A\&A, 556, A104

Purcell, J. D., \& Koomen, M. J. 1962, Report of NRL Progress (Washington, DC: US GPO), 9

Renotte, E., Alia, A., Bemporad, A., et al. 2015, Proc. SPIE, 9604, 96040A

Rougeot, R., \& Aime, C. 2018, A\&A, 612, A80

Rougeot, R., Flamary, F., Galano, D., \& Aime, C. 2017, A\&A, 590, A2

Rougeot, R., Aime, C., Baccani, C., et al. 2018, Proc. SPIE, 10698, 106982 T

Shestov, S., \& Zhukov, A. N. 2018, A\&A, 612, A82

Vanderbei, R. J., Kasdin, N. J., \& Cady, E. 2007, ApJ, 665, 794

van Hamme, W. 1993, AJ, 106, 2096

Venet, M., Bazin, C., Koutchmy, S., \& Lamy, P. 2010, ICSO 2010

Verroi, E., Frassetto, F., \& Naletto, G. 2008, J. Opt. Soc. Am. A, 25, 1 Zhukov, A. N., Veselovsky, I. S., Koutchmy, S., \& Delanné, C. 2000, A\&A, 353, 


\section{Appendix A: Two dimension image of the PSF}

In this appendix, we show the two dimension image of the PSF $\left|\Psi_{B, 0,0}(x, y)\right|^{2}$ from the on-axis point source in the focal plane of the telescope, that us, plane $B$. The area is $5.3 \operatorname{arcmin} \times 2 \operatorname{arcmin}$, and the center of the field-of-view is on the left boundary of the image.
In panel a of Fig. A.1 we plots the ideal PSF, that is, the classical Airy disk pattern, and in panel b the same PSF including the speckles, induced by the wave front error from the surface roughness in plane $A$. We enhanced these effects by increasing the roughness to $\sigma_{r}=21 \mathrm{~nm}$, for illustration purpose. Here, the disturbances of the PSF start being visible from about 4 arcmin.

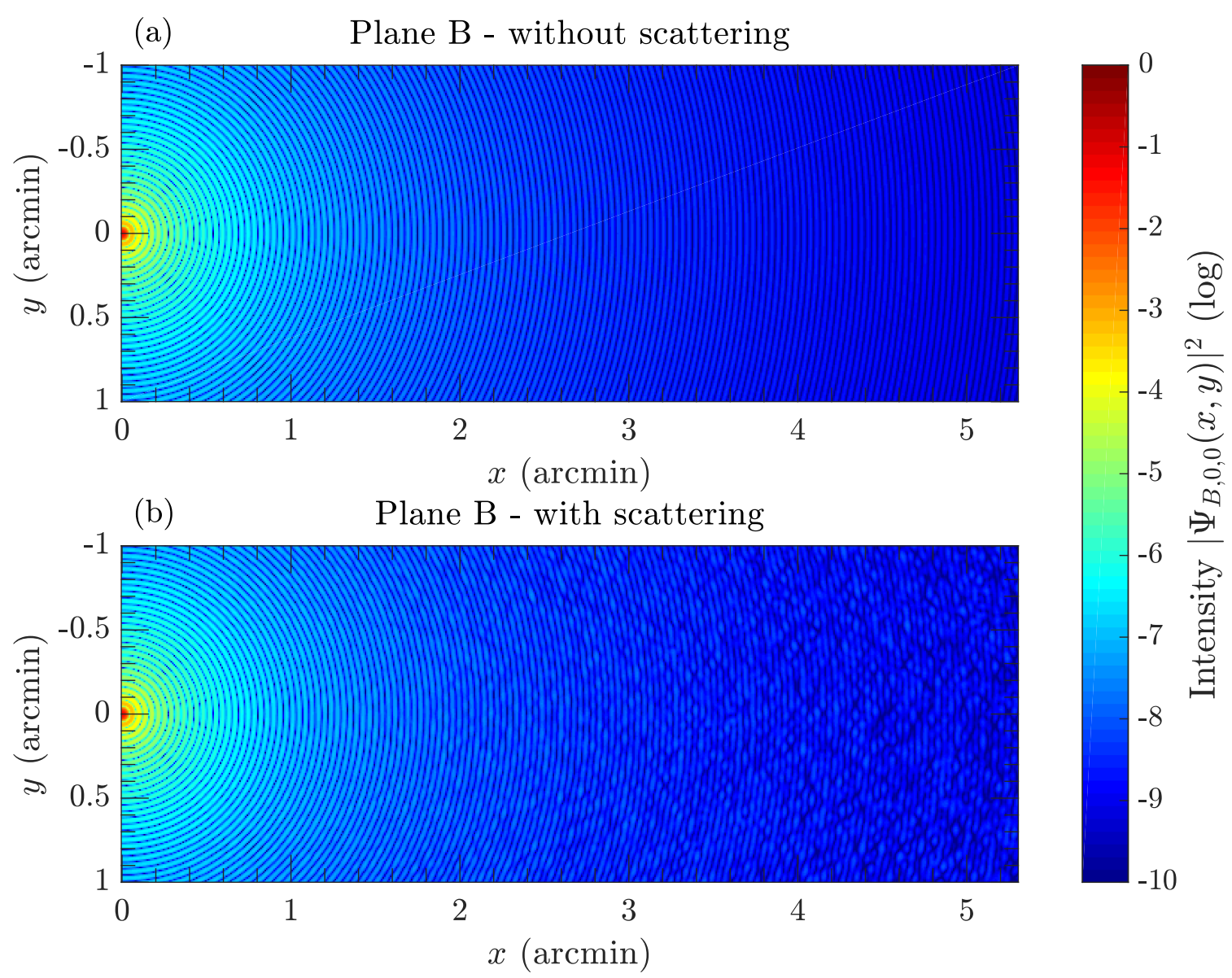

Fig. A.1. Two dimension PSF intensity $\left|\Psi_{B, 0,0}(x, y)\right|^{2}$ from the on-axis point source in plane $B$, in logarithmic scale. The area is 5.3 arcmin $\times 2$ arcmin. Panel $a$ : ideal PSF, without scattering. Panel $b$ : PSF with speckles, visible from 4 arcmin, because of the wave front error from surface roughness in plane $A$. 